\title{
Related Properties of Quantum Measurements
}

\author{
Qi Han, Ziqiang Lu*, Yanan Han
}

College of Mathematics and Statistics, Northwest Normal University, Lanzhou, 730070, Gansu P. R. China

DOI: $10.36347 /$ sipms.2020.v07i08.001

| Received: 26.07.2020 | Accepted: 04.08.2020 | Published: 08.08.2020

*Corresponding author: Ziqiang Lu

Abstract

Review Article

In this article, we elaborate the mathematical framework of quantum mechanics, we obtaine relevant results about quantum measurements, and find that for an arbitrary 2-qubit state, the order of the partial measurement in computational basis only affects the probability of obtaining the measurement result.

Keywords: quantum mechanics, quantum measurements, probability.

Copyright @ 2020: This is an open-access article distributed under the terms of the Creative Commons Attribution license which permits unrestricted use, distribution, and reproduction in any medium for non-commercial use (NonCommercial, or CC-BY-NC) provided the original author and source are credited.

\section{INTRODUCTION}

Quantum measurement is the main way to obtain quantum information which plays an important role in the information industry [6]. For the quantum measurement a more complete approach, see [1]. Quantum computing is the basis of the quantum measurement which the specific introduction and applications can be see [2, 3, 4, 7]. In recent years, Davis [8] discovered that quantum measurement may also affect the development of human perception and consciousness.

Quantum measurement is mainly to read the final quantum state after the closed quantum system interacts with the physical quantity, which makes the quantum state change. In generally, the quantum measurement will irreversibly change the quantum state.

This article mainly discusses the projective measurement and the measurement in computational basis. Under the premise of following postulates of quantum mechanics, for an arbitrary two-qubit state, the order of partial measurement in computational basis will affect the probability of obtaining the measurement result, but it will not affect the final measurement result.

We briefly summarize the contents of this paper. In section 2, we elaborate the mathematical framework of quantum mechanics. In section 3, we focus on the projective measurement and the partial measurement in computational basis, moreover, we obtain the important result that for an arbitrary twoqubits state, the order of the partial measurement in computational basis in that state only affects the probability of obtaining the corresponding result. In section 4 , we made a brief summary.

\section{Preliminary knowledge of quantum mechanics}

Quantum mechanics provides the mathematical framework for the development of physical theory, and its four postulates relate the physical world to the mathematical description of quantum mechanics.

Qubits as the most basic quantum mechanical system. A qubit has a two-dimensional state space. Let $|0\rangle$ and $|1\rangle$ constitute a standard orthogonal basis of the state space, then any vectors in the state space can be written

$$
|\psi\rangle=a|0\rangle+b|1\rangle
$$

Where $a$ and $b$ are complex numbers and

$$
|a|^{2}+|b|^{2}=1 \text {, that is }\langle\psi \mid \psi\rangle=1 \text {. }
$$

When we consider the composite quantum system composed of two or more different isolated physical systems. The composite system postulate gives a description method.

Definition 1. [5] The postulate of composite systems states that the state space of a composite system is the states of $n$ isolated quantum systems, the state of the composite system is

$$
\left|\psi_{1}\right\rangle \otimes\left|\psi_{2}\right\rangle \otimes \cdots \otimes\left|\psi_{n}\right\rangle
$$


It is easy to see that the state space of a twoqubit is $\mathbb{C}^{4}=\mathbb{C}^{2} \otimes \mathbb{C}^{2}$.

Therefore, the basis vector is $|0,0\rangle,|0,1\rangle,|1,0\rangle,|1,1\rangle$,

Where $|0,0\rangle=|0\rangle \otimes|0\rangle .|0,1\rangle,|1,0\rangle,|1,1\rangle \quad$ Have a similar interpretation.

Definition 2. [6] In composite physical systems, a state that cannot be expressed as a tensor product of the states of each isolated system is called an entangled state.

We have assumed that the closed quantum system evolves according to the unitary operator, but if we want to observe the system to understand the internal situation, at this time, the closed quantum system interacts with the external macro environment, so it no longer obey the unitary evolution.

Proposition 1.[5] A projective measurement is described by a Hermitian operator $O$, called observable in the state space of the system being measured. The observable $O$ has a diagonal representation:

$$
O=\sum_{\lambda} \lambda P_{\lambda}
$$

Where $P_{\lambda}$ is the projector on the eigenspace of $O$ associated with the eigenvalue $\lambda$.

The possible results of measurement of the observableb $O$ are the eigenvalues $\lambda$. If the system state at the time of measurement is $|\psi\rangle$, the probability of obtaining the result $\lambda$ will be

$$
\| P_{\lambda}|\psi\rangle \|^{2} \text { or } p_{\lambda}=\left\langle\psi\left|P_{\lambda}\right| \psi\right\rangle \text {. }
$$

If the result of the measurement is $\lambda$, the state of the quantum system immediately after the measurement will be

$$
\left.\frac{1}{\sqrt{p_{\lambda}}}\left|P_{\lambda}\right| \psi\right\rangle .
$$

Since the possible outcomes of a measurement of observable $O$ obey a probability distribution, we can define the expected value of a measurement as

$$
\langle O\rangle=\sum_{\lambda} p_{\lambda} \lambda
$$

The standard deviation as

$$
\Delta O=\sqrt{\left\langle O^{2}\right\rangle-\langle O\rangle^{2}} .
$$

\section{Measurement and partial measurement in} computational basis

The computational basis of space $c^{2}$ is the set $\{|0\rangle,|1\rangle\}$. For one qubit, the observable of the measurement in the computational basis is Pauli matrix $Z$, whose spectral decomposition is $\mathrm{Z}=(+1) \mathrm{P}_{+1}+(-1) \mathrm{P}_{-1}$,

Where $P_{+1}=|0\rangle\left\langle 0\left|, P_{-1}=\right| 1\right\rangle\langle 1|$

The possible results of the measurement are \pm 1 . Consider an observable $O$ with the following spectral decomposition

$$
O=\sum_{k=0}^{1} k|k\rangle\langle k| .
$$

Since the eigenvalues of $O$ are 0 and 1 , the above analysis holds if we replace +1 by 0 and -1 by 1 . With this new observable, there is a one-to-one correspondence in the nomenclature of the measurement result and the final state. If the result is 0 , the state after the measurement is $|0\rangle$. If the result is 1 , the state after the measurement is $|1\rangle$.

The computational basis of the Hilbert space of $n$ qubits in decimal notation is the set $\left\{|0\rangle, \cdots\left|2^{n}-1\right\rangle\right\}$. Generalizing to $n$ qubits, we have the following proposition.

Proposition 2.[5] The measurement in the computational basis is associated with observable

$$
O=\sum_{k=0}^{2^{n}-1} k|k\rangle\langle k| .
$$

A generic state of $n$ qubits is given by

$$
|\psi\rangle=\sum_{k=0}^{2^{n}-1} a_{k}|k\rangle
$$

where amplitudes $a_{k}$ satisfying the constraint

$$
\sum_{k}\left|a_{k}\right|^{2}=1 \text {. }
$$

The measurement result is an integer value $k$ in the range $0 \leq k \leq 2^{n}-1$ with a probability distribution given by

$$
p_{k}=\left\langle\psi\left|P_{k}\right| \psi\right\rangle=\left|a_{k}\right|^{2} \text {. }
$$


The $n$ qubit state immediately after the measurement is

$$
\frac{1}{\sqrt{p_{k}}} P_{k}|\psi\rangle \square|k\rangle \text {. }
$$

Proposition 3. For a given state $|\psi\rangle$, the expected value of an arbitrary measurement observed at the state has the following expression:

$$
\langle O\rangle=\langle\psi|O| \psi\rangle .
$$

Proof. By (1), (2), (3), we have $\langle O\rangle=\langle\psi|O| \psi\rangle$

Proposition 4. If the physical system is in a state $|\psi\rangle$ that is an eigenvector of $O$, then $\Delta O=0$.

Proof. From the definition of standard deviation, just prove

$$
\left\langle O^{2}\right\rangle=\langle O\rangle^{2}
$$

By Proposition 3,

$$
\left\langle O^{2}\right\rangle=\left\langle\psi\left|O^{2}\right| \psi\right\rangle=\left\langle\psi \mid\left(\sum_{\lambda} \lambda P_{\lambda}\right)^{2} \psi\right\rangle
$$

Since $P_{\lambda}$ is a projection operator,

$$
P_{\lambda}^{2}=P_{\lambda}=P_{\lambda}^{\dagger}
$$

where $†$ means hermite conjugate. We have

$$
\left\langle O^{2}\right\rangle=\left\langle\psi \mid \sum_{\lambda} \lambda^{2} P_{\lambda}^{\dagger} P_{\lambda} \psi\right\rangle
$$

And since $|\psi\rangle$ is an eigenvector, suppose the correspondence eigenvalue is $\lambda_{i}$, we have

$$
\left\langle O^{2}\right\rangle=\left\langle\psi \mid \lambda_{i}^{2} P_{\lambda_{i}}^{\dagger} P_{\lambda_{i}} \psi\right\rangle=\lambda_{i}^{2} .
$$

Similarly,

$$
\langle O\rangle^{2}=(\langle\psi|O| \psi\rangle)^{2}=\left(\left\langle\psi \mid \sum_{\lambda} \lambda P_{\lambda} \psi\right\rangle\right)^{2}=\left(\left\langle\psi \mid \lambda_{i} P_{\lambda_{i}}^{\dagger} P_{\lambda_{i}} \psi\right\rangle\right)^{2}=\lambda_{i}^{2}
$$

Thus $\Delta O=0$.

Proposition 5. Suppose that the physical system is in generic state $|\psi\rangle$, then $\sum_{\lambda} p_{\lambda}^{2}=1$ to an observable $O$ if and only if $\Delta O=0$.

Proof. By $\sum_{\lambda} p_{\lambda}^{2}=1=\sum_{\lambda} p_{\lambda}$ and Non-negativity of probability, we have $p_{\lambda_{k}}=1$.

Suppose $|\psi\rangle=\sum_{k=0}^{2^{n}-1} a_{k}|k\rangle$, we have

$$
\langle O\rangle^{2}=\left(\sum_{\lambda} p_{\lambda} \lambda\right)^{2}=\left(\sum_{\lambda}\left\langle\psi\left|P_{\lambda}\right| \psi\right\rangle \lambda\right)^{2}=\left(\left|a_{k}\right|^{2}\left\langle k\left|P_{\lambda_{k}}\right| k\right\rangle \lambda_{k}\right)^{2}=\left|a_{k}\right|^{2} \lambda_{k}^{2}
$$

Similarly,

$$
\left\langle O^{2}\right\rangle=\left\langle\psi\left|O^{2}\right| \psi\right\rangle=\left\langle\psi\left|\left(\sum_{\lambda} \lambda P_{\lambda}\right)^{2}\right| \psi\right\rangle=\left\langle\psi\left|\sum_{\lambda} \lambda^{2} P_{\lambda}^{\dagger} P_{\lambda}\right| \psi\right\rangle=\left|a_{k}\right|^{2} \lambda_{k}^{2}
$$

Thus $\Delta O=0$. 
By $\Delta O=0$ and $\sum_{\lambda} p_{\lambda}=1$, we have

$$
\sum_{\lambda} p_{\lambda}=1=p_{\lambda_{k}}=\sum_{\lambda} p_{\lambda}^{2}
$$

The term measurement in the computational basis of $n$ qubits implies a measurement of all $n$ qubits. However, it is possible to perform a partial measurement; the result in this case is not necessarily a state of the computational basis.

Definition 3. [5] If a system composed of subsystems $A$ and $B$, a partial measurement is a measurement of an observable of the type $O_{A} \otimes I_{B}$ or $O_{B} \otimes I_{A}$, where $O_{A}$ and $O_{B}$ are an observable of system $A$ and $B$, respectively. $I_{A}$ and $I_{B}$ are the identity operator of system $A$ and $B$, respectively. Physically, this means that the measuring apparatus interacted only with the subsystem.

Proposition 6. [5] A composite quantum system composed of two isolated quantum systems $A$ and $B$, where the state space of system $A$ is $m$ dimensional Hilbert space and the state space of system $B$ is $n$-dimensional Hilbert space, then the computation basis is the set

$$
\left\{|i, j\rangle: 0 \leq i \leq 2^{m}-1,0 \leq j \leq 2^{n}-1\right\}
$$

A generic state will be represented by

$$
|\psi\rangle=\sum_{i=0}^{2^{m}-1} \sum_{j=0}^{2^{n}-1} a_{i j}|i, j\rangle .
$$

Suppose we measure observable $O_{A} \otimes I_{B}$, where

$$
O_{A}=\sum_{k=0}^{2^{m}-1} k P_{k}
$$

The probability of obtaining value $0 \leq k \leq 2^{m}-1$ is

$$
p_{k}=\left\langle\psi\left|\left(P_{k} \otimes I\right)\right| \psi\right\rangle=\sum_{j=0}^{2^{n}-1}\left|a_{k j}\right|^{2} .
$$

The set $\left\{p_{0}, \cdots, p_{2^{m}-1}\right\}$ is a probability distribution and therefore satisfies

$$
\sum_{k=0}^{2^{m}-1} p_{k}=1
$$

If the measurement result is $k$, the state immediately after the measurement will be

$$
\frac{1}{\sqrt{p_{k}}}\left(P_{k} \otimes I\right)|\psi\rangle=\frac{1}{\sqrt{p_{k}}}|k\rangle\left(\sum_{j=0}^{2^{n}-1} a_{k j}|j\rangle\right) \text {. }
$$

Proposition 7. For an arbitrary 2-qubit state, the order of the partial measurement only affects the probability of obtaining the corresponding result of the measurement.

Proof. Suppose an arbitrary state is

$$
|\psi\rangle=a|0,0\rangle+b|0,1\rangle+c|1,0\rangle+d|1,1\rangle \text {, }
$$

Where $a, b, c, d$ are constants. Using Pauli $Z$ matrix for a partial measurement.

For observable $Z_{1}$, when the measurement result is 0 or 1 , we have the probability of the measurement result is

$$
p_{0}=|a|^{2}+|b|^{2} \text { or } p_{1}=|c|^{2}+|d|^{2},
$$

The state immediately after the measurement will be

$$
\frac{a}{\sqrt{|a|^{2}+|b|^{2}}}|0,0\rangle+\frac{b}{\sqrt{|a|^{2}+|b|^{2}}}|0,1\rangle \text { or } \frac{c}{\sqrt{|c|^{2}+|d|^{2}}}|1,0\rangle+\frac{d}{\sqrt{|c|^{2}+|d|^{2}}}|1,1\rangle \text {. }
$$

After measuring $Z_{1}$, we measure $Z_{2}$, when the measurement result is $00,10,01,11$, respectively, we have the probability of the measurement result is

$$
p_{00}=\frac{|a|^{2}}{|a|^{2}+|b|^{2}}, p_{10}=\frac{|c|^{2}}{|a|^{2}+|d|^{2}}, p_{01}=\frac{|b|^{2}}{|a|^{2}+|b|^{2}}, p_{11}=\frac{|d|^{2}}{|a|^{2}+|d|^{2}},
$$

Respectively. The state immediately after the measurement will be

$$
|0,0\rangle,|1,0\rangle,|0,1\rangle,|1,1\rangle, \text { respectively. }
$$


We reverse the order of the measurement, for observable $Z_{2}$, when the measurement result is 0 or 1 , we have the probability of the measurement result is

The state immediately after the measurement will be

$$
p_{0}=|a|^{2}+|c|^{2} \text { or } p_{1}=|b|^{2}+|d|^{2},
$$

$$
\frac{a}{\sqrt{|a|^{2}+|c|^{2}}}|0,0\rangle+\frac{c}{\sqrt{|a|^{2}+|c|^{2}}}|1,0\rangle \text { or } \frac{b}{\sqrt{|b|^{2}+|d|^{2}}}|0,1\rangle+\frac{d}{\sqrt{|b|^{2}+|d|^{2}}}|1,1\rangle \text {. }
$$

After measuring $Z_{2}$, we measure $Z_{1}$, when the measurement result is $00,10,01,11$, respectively, we have the probability of the measurement result is

$$
p_{00}=\frac{|a|^{2}}{|a|^{2}+|c|^{2}}, p_{10}=\frac{|c|^{2}}{|a|^{2}+|c|^{2}}, p_{01}=\frac{|b|^{2}}{|b|^{2}+|d|^{2}}, p_{11}=\frac{|d|^{2}}{|b|^{2}+|d|^{2}},
$$

Respectively. The state immediately after the measurement will be

$$
|0,0\rangle,|1,0\rangle,|0,1\rangle,|1,1\rangle \text {, respectively. }
$$

We found that after reversing the order of the partial measurement, the measurement results are consistent. However, the probability of obtaining the same measurement results is inconsistent.

\section{CONCLUSIONS}

In this article, we have obtained some properties about quantum measurement by postulates of quantum mechanics. The main result is that the order of the partial measurement in computational basis only affects the probability of obtaining the measurement results.

\section{REFERENCES}

1. Diu B, Cohen-Tannoudji C, Laloe F. Quantum Mechanics. Wiley-Interscience, New York.2006.

2. Kaye P, Laflamme R, Mosca M. An introduction to quantum computing. Oxford university press; 2007.

3. Mermin ND. Quantum computer science: an introduction. Cambridge University Press; 2007 Aug 30.

4. Rieffel EG, Polak WH. Quantum computing: A gentle introduction. MIT Press; 2011 Mar 4.

5. Howard B. Nicolas Gisin.:Quantum Science and Technology[J]. Springer New York Heidelberg Dordrecht London. 2013.

6. Nielsen MA, Computation IC. Quantum Information Cambridge University Press.2000.

7. $\mathrm{Li} \mathrm{YL}, \mathrm{Zu} \mathrm{CJ}$, Wei DM. Enhance quantum teleportation under correlated amplitude damping decoherence by weak measurement and quantum measurement reversal. Quantum Information Processing. 2019 Jan 1;18(1):2.

8. Davis JJ. Indeed, Quantum Measurement may just play a part in human brain dynamics that together with the soul may affect human perception and consciousness development. Activitas Nervosa Superior. 2019 May 25:1-6. 